\title{
Erratum: Onset of random matrix behavior in scrambling systems
}

\author{
Hrant Gharibyan, ${ }^{a}$ Masanori Hanada, ${ }^{a, b, c, d}$ Stephen H. Shenker ${ }^{a}$ and Masaki Tezuka ${ }^{e}$ \\ ${ }^{a}$ Stanford Institute for Theoretical Physics, Stanford University, \\ Stanford, CA 94305, U.S.A. \\ ${ }^{b}$ Yukawa Institute for Theoretical Physics, Kyoto University, \\ Kyoto 606-8502, Japan \\ ${ }^{c}$ The Hakubi Center for Advanced Research, Kyoto University, \\ Kyoto 606-8501, Japan \\ ${ }^{d}$ Department of Physics, University of Colorado, \\ Boulder, Colorado 80309, U.S.A. \\ e Department of Physics, Kyoto University, \\ Kyoto 606-8502, Japan \\ E-mail: hrant@stanford.edu, hanada@yukawa.kyoto-u.ac.jp, \\ sshenker@stanford.edu, tezuka@scphys.kyoto-u.ac.jp
}

ERRATUM TO: JHEP07(2018)124

ArXiv EPrint: 1803.08050 
We have found an error in section 6 of this paper. In that section we gave a heuristic argument estimating the ramp time of Hamiltonian systems by assuming that the slowest decay in eq. (105) was that of simple operators. However that argument has an error. ${ }^{1}$ Two point functions of operators that couple to the Hamiltonian have subleading terms that do not decay in time because of energy conservation. ${ }^{2}$ They remain constant for all times. These terms are small, but are much larger than the ramp time value and so need to be controlled to make a reliable estimate.

In fact we believe that these terms almost exactly cancel at the ramp time. A reliable calculation of the ramp time has been done for Hamiltonian SYK in [1] using the collective field formalism. The result there is consistent with the intuition described in section 6 . We have modified section 6 in the version of the paper posted on the ArXiv to reflect this. To be clear, the results and analysis of the rest of the paper are completely independent from this section and the only other changes that have been are to the parts of the Abstract, Introduction and Discussion that referred to section 6 .

Open Access. This article is distributed under the terms of the Creative Commons Attribution License (CC-BY 4.0), which permits any use, distribution and reproduction in any medium, provided the original author(s) and source are credited.

\section{References}

[1] P. Saad, S.H. Shenker and D. Stanford, A semiclassical ramp in SYK and in gravity, arXiv: 1806.06840 [INSPIRE].

\footnotetext{
${ }^{1}$ We thank Alex Altland, Dmitry Bagrets and Alexei Kitaev for pointing this problem out to us.

${ }^{2}$ An example of such a two point function in SYK is $\left\langle\psi_{1} \psi_{2} \psi_{3} \psi_{4}(t) \psi_{1} \psi_{2} \psi_{3} \psi_{4}(0)\right\rangle_{\beta}$ that has a $1 / N^{3}$ suppressed term that doesn't decay in time, whereas leading term decays exponentially.
} 